\title{
Lucky labelling and Radio labelling of Certain Leaf Vein Graphs
}

\author{
Kins Yenoke ${ }^{1}$, Catherine Saranya $\mathrm{A}^{2}$, Maria Jenifer $\mathrm{V}^{2}$, Lilly Shamini $\mathrm{Y}^{2}$ \\ Assistant Professor, Department of Mathematics, Loyola College, Chennai, India ${ }^{1}$ \\ PG student, Department of Mathematics, Loyola College, Chennai, India ${ }^{2}$
}

\begin{abstract}
Let $G(V, E)$ be a graph with vertex set $V$ and edge set $E$. Let c be a labelling defined in $G$. Define the sum of neighbourhood of vertex $v$ by $s(v)=\sum_{u \in N(v)} c(u)$, where $N(v)$ denotes the open neighbourhood of $v \in V$. A labelling $c$ is a lucky labelling if $s(u) \neq s(v)$ for all $(u, v) \in E(G)$. The lucky number of $G$ is denoted by $\eta(G)$, is the least positive integer $k$ such that $G$ has alucky labelling with $\{1,2 \ldots k\}$ as the set of labels. A radio labelling of a connected graph $G$ with diameter $d$ is an injection ffrom the vertices of $G$ to the set of natural numbers such that $d(u, w)+|f(u)-f(w)| \geq 1+d$, for every pair of vertices $u$ and $w$ of $G$. The radio number of $\boldsymbol{f}$ denoted by $r n(f)$, is the maximum number assigned to any vertex of $G$. The radio number of $\boldsymbol{G}$, denoted byrn $(G)$, is the minimum value of $r n(f)$, taken over all labelling $f$ of $G$. In this paper we have constructed the leaf vein graphs, namely, Jasmine leaf vein graph, Ficus religiosa leaf vein graph and Hibiscus Rosa-Sinensisleaf vein graph. Also, we have determined the Lucky number and Radio number for such graphs.
\end{abstract}

KEYWORDS: Labelling, Lucky number, Radio labelling, Radio number, Jasmine leaf vein graph, Ficus religiosa leaf vein graph, Hibiscus Rosa-Sinensis leaf vein graph.

\section{INTRODUCTION}

Neural networks are one of the most popular machine learning algorithms for plant leaf classification. In 2018 Tan Kiet Nguyen Thanh[8] et.al. studied depth learning with convolutional neural network for leaves classifier based on shape of leaf vein. Nowadays, depth learning with Convolution Neural Networks (CNN) has become a wellknown method in object recognition task to generate descriptions and classify learned features and gradually took over. There aremany CNN applied studies on leaves recognition and classifier task that mainly use the existing CNN architecture and pre-trained models. The plants identification systems were implemented in different ways by different authors. Several researches [7, 10] used neural networks and the others used matching strategies to study their research problems. As we are going deeper into CNNs and graphs, we found the keys of CNNs: local connection, shared weights and the use of multi-layer [9]. These are also of great importance in solving problems of graph domain, because graphs are the most typical locally connected structure.

The applications of artificial neural networks for many difficult problems of graph theory, especially NP-complete problems, and the applications of graph theory to artificial neural networks are discussed. For example, graph theory is used to study the pattern classification problem on the discrete type feedforward neural networks, and the stability analysis of feedback artificial neural networks etc. This paper aims to study the different vein patterns and their graph structure based on the primary, secondary and tertiary veins. Also, the graph labelling problems such as radio labelling problem and lucky labelling problems were discussed for the leaf vein graphs.

The use of graph theory to study the Channel Assignment Problem and related problems dates back at least to 1970 . In 2001 Chartrand et al. [3] were motivated by regulations for channel assignments of FM radio stations to introduce radio labellings of graphs. For a connected graph $G$ of diameter $d$, a radio labelling of $G$ is an injection $f$ from the vertices of $G$ to the natural numbers such that $d(u, w)+|f(u)-f(w)| \geq 1+d \forall u, v \in V(G)$. The radio number of $f$ denoted by $r n(f)$, is the maximum number assigned to any vertex of $G$. The radio number of $G$, denoted byrn $(G)$, is the minimum value of $r n(f)$, taken over all labelling $f$ of $G$. The upper and lower bounds for the radio numbers of different networks and graphs were studied by several authors. The radio number for square of paths and of cycles was investigated by Liu et al.Fernandez et al. [5]computed $r n\left(G_{n}\right)=4 n+2, n \geq 4$, where $G_{n}$ is the $n$-gear graph. Kins Yenoke et.al. [6] obtained the upper and lower bounds for the radio number of enhanced mesh $E N(n, n)$.

In recent years, the lucky labeling of graphs was studied by A. Ahai et. al. [1]. Suppose the vertices of a graph $G$ were labeled arbitrarily by positive integers and let $\mathrm{s}(v)$ denote the sum of labels over all neighbors of vertex $v$. A labeling is lucky if the function s is a proper coloring of $G$, that is, if we have $s(u) \neq s(v)$ if $u$ and $v$ are adjacent vertices. 


\title{
| e-ISSN: 2319-8753, p-ISSN: 2320-6710| www.ijirset.com | Impact Factor: 7.512|
}

\author{
\|Volume 9, Issue 6, June 2020\|
}

The least positive integer $k$ for which a graph $G$ has a lucky labeling from the set $\{1,2 \ldots k\}$ is the lucky number of $G$, denoted by $\eta(G)$.

\section{LEAF VEIN GRAPHS}

In this section we have constructed the certain leaf vein graphs such as Jasmine leaf vein graph, Ficus religiosa vein leaf graph and Hibiscus Rosa-Sinensis vein leaf graph.

A leaf is an organ of a vascular and is the principal lateral appendage of the stem. Some leaves have a petiole, which attaches the leaf to the stem; leaves that do not have petioles are directly attached to the plant stem and are called sessile leaves. The arrangement of veins in a leaf is called the venation pattern; monocots have parallel venation, while dicots have reticulated venation. Veins provide support for the leaf and transport both water and minerals (via xylem) and food energy (via phloem) through the leaf and on to the rest of the plant.

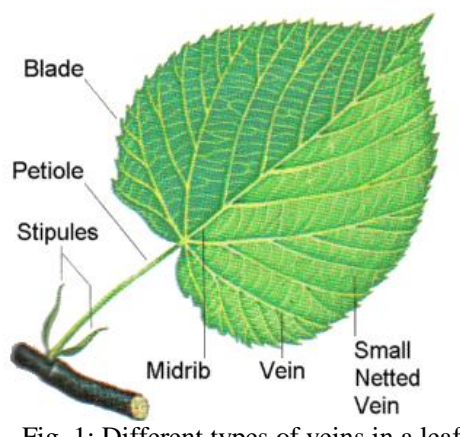

Veins are composed of xylem and phloem cells embedded in parenchyma, sometimes sclerenchyma, and surrounded by bundle sheath cells. The vein xylem transports water from the petiole throughout the lamina mesophyll, and the phloem transports sugars out of the leaf to the rest of the plant. Leaf venation systems vary strongly across major plant lineages, with many early groups having dichotomously branching, open systems, but reticulation evolved frequently. Angiosperms have greatest diversity in vein structure but share key architectural elements, that is, a hierarchy of vein orders forming a reticulate mesh.

Typically, there are three orders of lower-order veins, known as 'major veins', often ribbed with sclerenchyma. One or more first-order veins run from the petiole to the leaf apex, with second-order veins branching at intervals, and third-order veins branching between. The major veins can be distinguished from minor veins, typically present only in angiosperms, which include up to four additional orders of smaller, reticulate higher-order veins. Major and minor veins can be distinguished by their distinct timing of formation, and differences in gene expression during development, sizes and branching in the mature leaf, and in cross-sectional anatomy. In several lineages, particularly the monocotyledons, a grid-like 'striate' venation is typical, including several orders of longitudinal veins of different sizes, with small transverse veins connecting them.
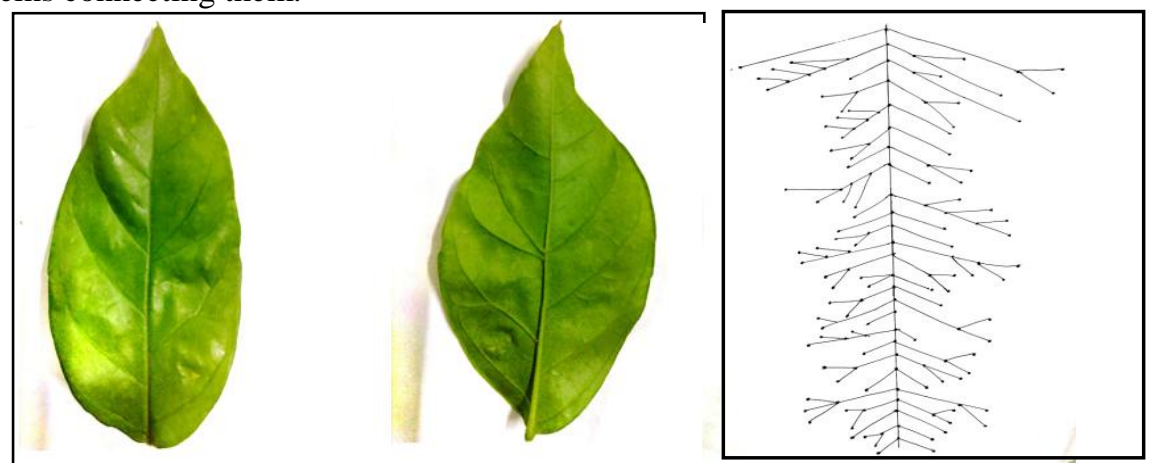

Fig. 2: Jasmine Plant leaf front view, rear view and its veins in graph structure

The leaf vein graph construction is the first step in this research. The graph was constructed with the help of a fullgrown matured leaf. A leaf was taken from a tree of normal growth without any restrictions. Due care was taken to ensure that the leaf was not infected by any pest. The leaf thus obtained was dried for 3-4 days, until the veins were exposed, then the primary, secondary and tertiary veins were marked. The junction of each vein was taken as vertices 


\section{||Volume 9, Issue 6, June 2020\|}

and the vein in itself was taken as the edge. The steps were repeated for all veins. This way we constructed the leaf vein graphs.

Remark 1:The number of vertices and edges of Jasmine leaf vein graph are 158 and 157 respectively. It's a planar graph with diameter 30 .

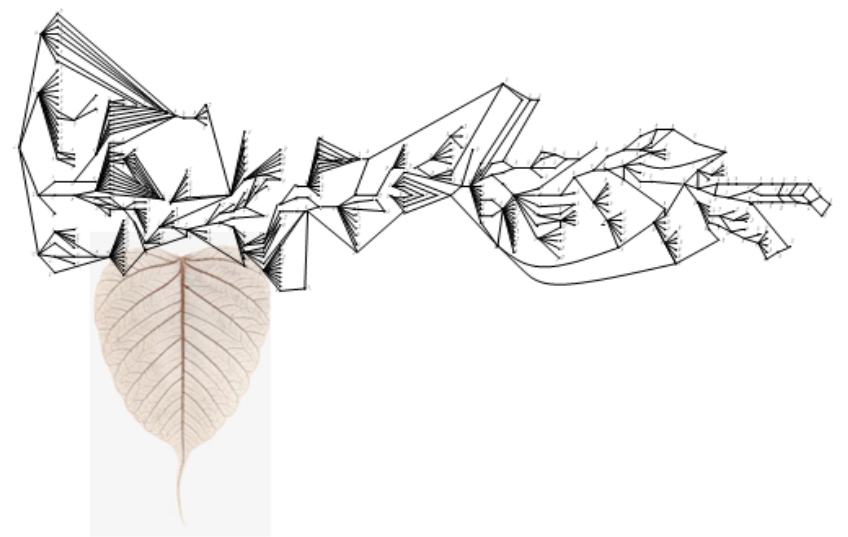

Fig. 3: Leaf of Ficus Religiosa and the constructed vein graph.

Remark 2: The number of vertices and edges of Religiosa leaf vein graph are 463 and 533 respectively. It's cyclic graph with diameter 49.

Remark 3:Rosa-sinensis leaf vein graph is a planar graph with 1488 vertices. The constructed graph is look like a tree structured graph with 70 levels. In this paper we name the vertices of Rosa-sinensis leaf vein graph as $V_{1}, V_{2} \ldots V_{1488}$ as shown in Fig. 4.

\section{RADIO NUMBER OF JASMINE LEAF VEIN GRAPH}

In this section we have determined the radio number of Jasmine leaf vein graph.

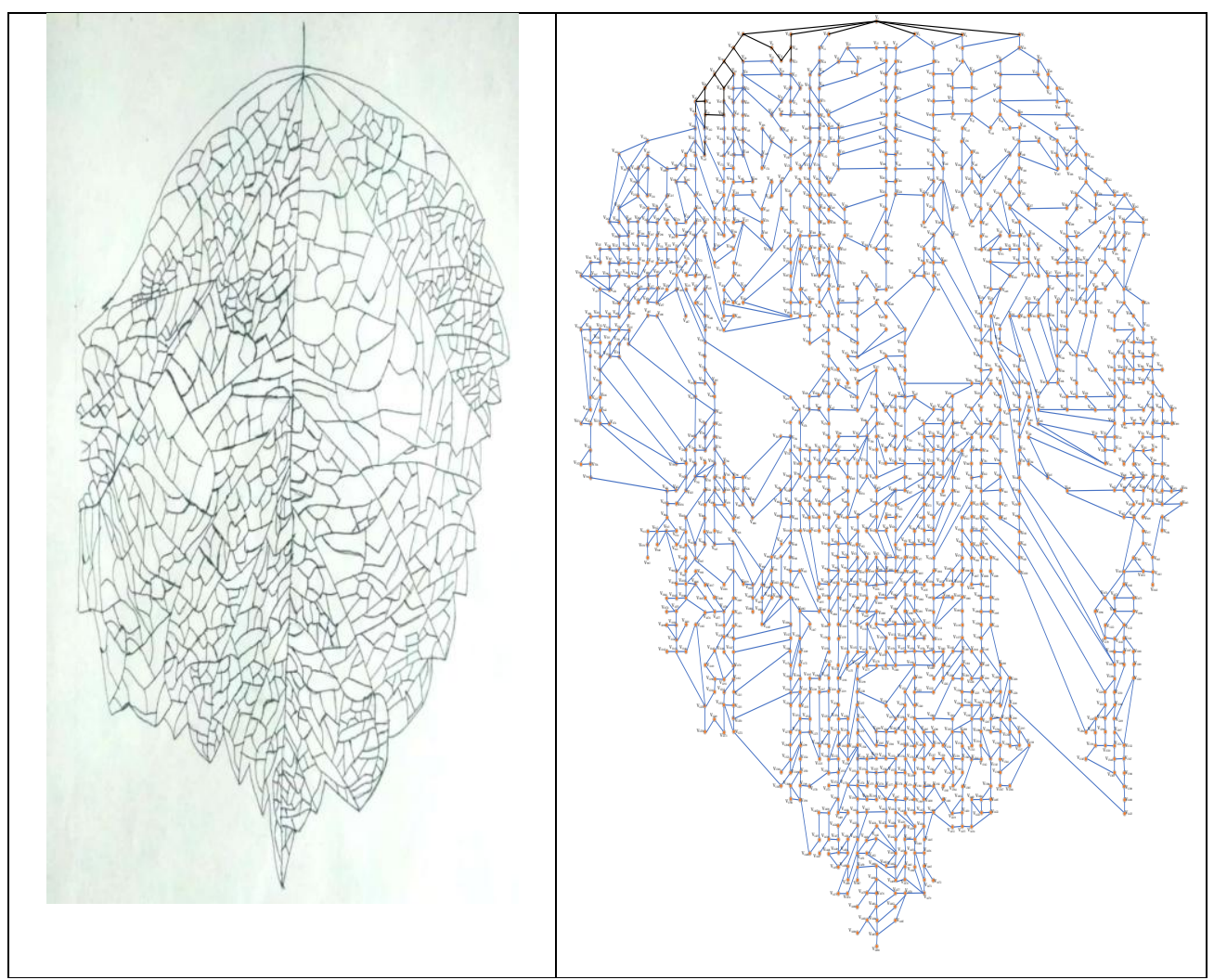

Fig. 4: Skeleton structure of Hibiscus Rosa-sinensis leaf and the constructed vein graph. 


\section{\|Volume 9, Issue 6, June 2020\|}

Theorem 3.1: Let $G$ be a Jasmine leaf vein graph, then the radio number of $G$ satisfies $r n(G) \leq 2772$.

Proof:First we name the vertices as $v_{1}, v_{2} \ldots v_{158}$ from top to down, that is, start from the first primary vein vertex and end with the last tertiary vein vertex as shown in Fig. 4.

Next we partition the vertex set into two disjoint sets $V_{1}=\left\{v_{1}, v_{2} \ldots v_{96}\right\}$ and $V_{2}=\left\{v_{97}, v_{98} \ldots v_{156}\right\}$.

Define a mapping $f: V(G) \rightarrow N$ as follows:

$$
\begin{gathered}
f\left(v_{i}\right)=30(i-1)+1, i=1,2 \ldots 96 \\
f\left(v_{96+i}\right)=30(i-1)+16, i=1,2 \ldots 62 . \text { See Fig. } 5 .
\end{gathered}
$$

Next, we verify the radio conditions $d(u, v)+|f(u)-f(v)| \geq d+1=31$ for all $u, v \in V(G)$.

Let $u$ and $v$ be any two vertices in $V(G)$.

Case 1: Suppose $u$ and $v$ lie in $V_{1}$, then $f(u)=30(t-1)+1$ and $f(v)=30(s-1)+1,1 \leq t \neq s \leq 96$. Also, the distance between them is atleast1. Hence $d(u, v)|+f(u)-f(v)| \geq 1+\mid 30(t-1)+1-30(s-1)-1) \mid=$ $1+|30(t-s)| \geq 31$, since $t \neq s$.

Case 2: Suppose $u, v \in V_{2}$, then $f(u)=30(t-1)+1, f(v)=30(s-1)+1,1 \leq t \neq s \leq 62$ and $d(u, v) \geq 1$. Therefore, $|f(u)-f(v)|+d(u, v) \geq 1+\mid 30(t-1)+1-30(s-1)-1)|=1+| 30 t-30+1-30 s+$ $30-1|=1+| 30(t-s) \mid) \mid \geq 31$, since $t \neq s$.

Case 3: Suppose $u \in V_{1}$ and $v \in V_{2}$, then the function values of $f$ are $f(u)=30(s-1)+1 ; 1 \leq s \leq 96$ and $f(v)=30(t-1)+1 ; 1 \leq t \leq 62$.

Case 3.1: When $s=t$, the distance between $u$ and $v$ is at least 16 and hence we get, $d(u, v)+|f(u)-f(v)| \geq 16+$ $15 \geq 31$.

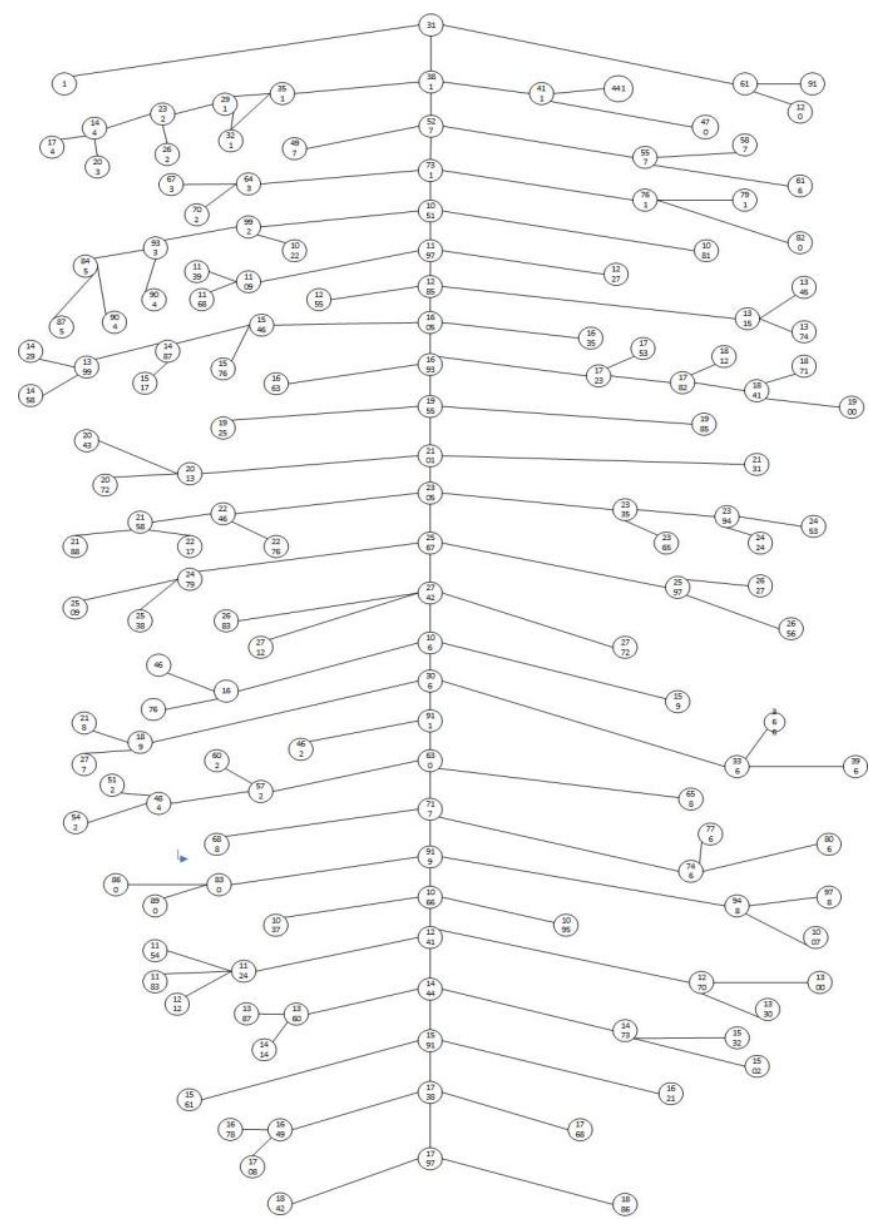

Fig. 5: A radio labelling of Jasmine leaf vein graph which attains the bound. 


\section{||Volume 9, Issue 6, June 2020\|}

Case 3.2:Ifs $\neq t$, then $d(u, v) \geq 16$.Also, $|f(u)-f(v)|+d(u, v) \geq 16+|30(s-1)+1-30(t-1)-1|=$ $16+|30(s-t)|>31$.

Thus, $d(u, v)+|f(u)-f(v)| \geq d+1=31 \forall u, v \in V(G)$. Therefore, the radio number of G satisfies $r n(G) \geq 2772$.

\section{LUCKY LABELLING OF ROSA-SINENSIS LEAF VEIN GRAPH AND RELIGIOSA LEAF VEIN GRAPH}

In this section we have obtained the lucky number of Rosa-Sinensis leaf vein graph and Religiosa leaf vein graph.

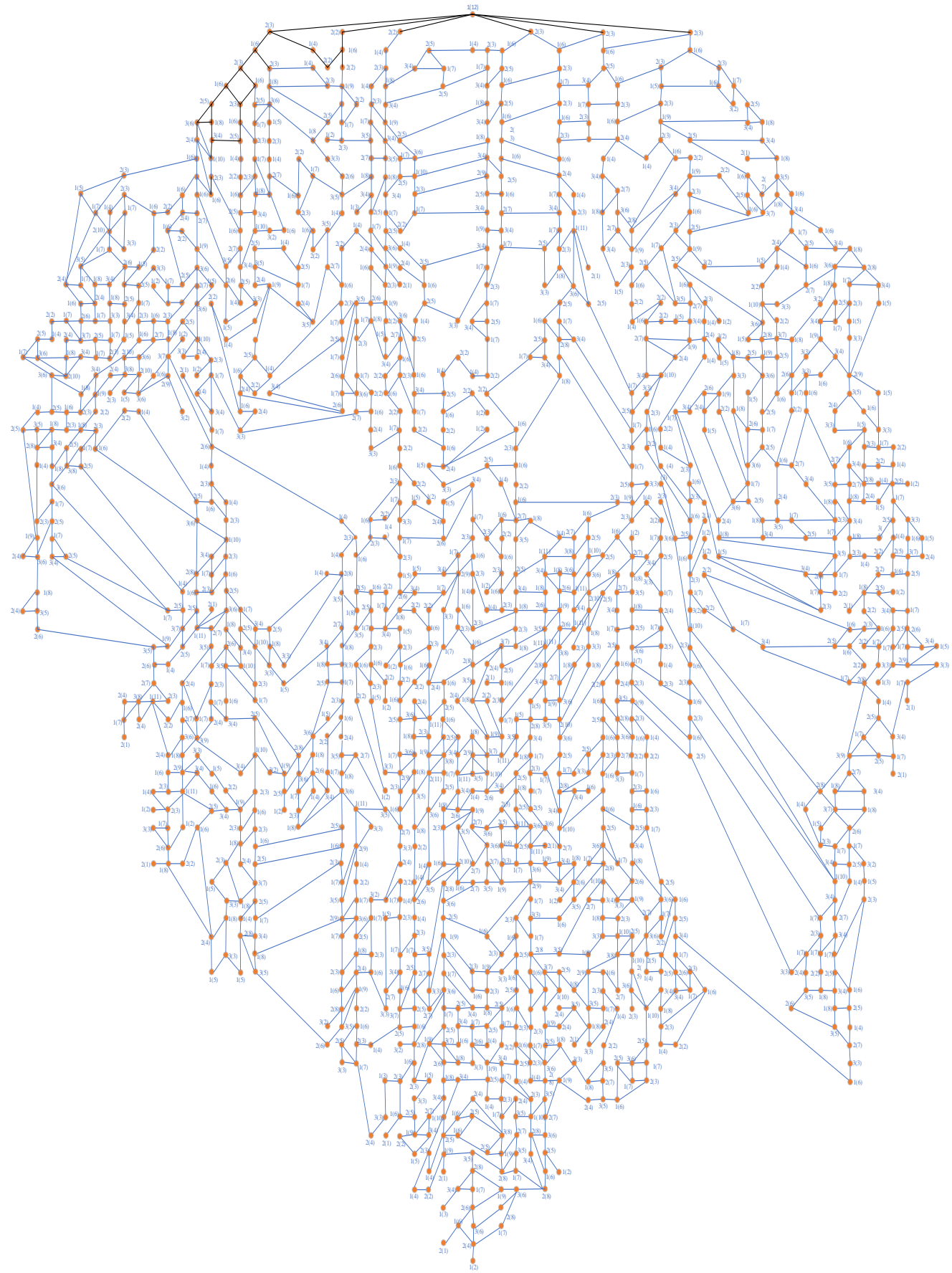

Fig. 6: A lucky labelling of Rosa-Sinensis leaf vein graph which attains the bound. 


\section{\|Volume 9, Issue 6, June 2020\|}

Theorem 4.1:Let $\mathrm{G}$ be aFicus Religiosa vein leaf graph, then the lucky number of $\mathrm{G}$ is $\eta(G)=2$.

Proof: First we start naming the vertices from the topmost primary vein vertex as $v_{1}$ and move down to the next level from left to right as $v_{2}, v_{3} \ldots$ till the last tertiary vein vertex $v_{463}$ is reached.Next, we partition the vertex set $V(G)=$ $\left\{v_{1}, v_{2} \ldots v_{463}\right\} \quad$ into two disjoint sets $V_{1}=$

$\left\{v_{3}, v_{5}, v_{27}, v_{28}, v_{30}, v_{56}, v_{79}, v_{104}, v_{105}, v_{106}, v_{107}, v_{108}, v_{112}, v_{110}, v_{116}, v_{189}, v_{215}, v_{219}, v_{220}, v_{229}, v_{234}, v_{262}, v_{268}, v_{269}, v_{313}, v_{319}, v_{36}\right.$ and $V_{2}=V-V_{1}$.

Define a mapping $f: V(G) \rightarrow N$ by $f(v)=1$, if $v \in V_{2}$ and $f(v)=2$, if $v \in V_{1}$.

From Fig. 3, we determined the sum of neighbourhood values of each vertex is either anyone of the value in the set $S=\{2,3, \ldots 16\}$. We can easily verify that $s(u) \neq s(v)$ for all $u, v \in V(G)$. Hence $f$ is a valid Lucky labelling.

Therefore $\eta(G) \leq 2$. But the lucky number of a path of length 2 is 2 .

Hence the lucky number of Ficus Religiosa vein leaf graph is at least 2 . Therefore, $\eta(G) \leq 2$, which proves the theorem.

Theorem 4.2: Let $G$ be a Rosa-Sinensis leaf vein graph, then the lucky number of $G$ is $\eta(G)=3$.

Proof: As the proof is similar to theorem 4.1, we left the proof to the reader.

\section{CONCLUSION}

In this paper we have constructed certain leaf vein graphs and apply certain graph labelling problems in it. The wonder of nature cannot be comprehended in a single study. Efforts have been taken to further extend the study to other family of tree leaves.

\section{REFERENCES}

1. A. Ahai, A. Dehghan, M. Kazemi, E. Mollaahmedi, "Computation of Lucky number of planar graphs is NP-hard", Information Processing Letters, Vol 112 (4), pp. 109-112, 2012.

2. S. Akhari, M. Ghanbari, R. Manariyat, S. Zare, 'On the lucky choice number of graphs", Graphs and Combinatorics, Vol 29, pp. 157-163,2013.

3. G. Chartrand, D. Erwin, and P. Zhang: A graph labelling problem suggested by FM channel restrictions, Bull. Inst. Combin. Appl., 43 (2005), 43-57.

4. Czerwinski, Sebastian, Grytczuk, Jaroslaw, Elazny and Wiktor, "Lucky labelling of graphs", Inf. Process. Lett. Vol.109 (18), pp. 1078-1081, 2009.

5. C. Fernandez, A. Flores, M. Tomova and Cindy Wyels, The Radio Number of Gear Graphs, http ://arxiv.org/P Scache/arxiv/pdf /0809/0809.2623v1.pdf.

6. Kins Yenoke, P. Selvagopal, K.M Baby Smitha, Ronald Cranston, "Bounds for the Radio Number of Mesh Derived Architecture", International Journal of Innovative Research in Science, Engineering and Technology, Vol. 9 (6), pp. 4806-4810, 2020.

7. Y. LeCun, Y. Bengio, and G. Hinton, “Deep learning,” Nature 2015, vol. 521, pp. 436-442, 2015.

8. Quoc Bao Truong, Quoc Dinh Truong, Hiep Huynh Xuan, "Depth Learning with Convolutional Neural Network for Leaves Classifier Based on Shape of Leaf Vein” Lecture Notes in Computer Science, Vol. 10751, pp. 565$575,2018$.

9. Wu, S. G., Bao, F. S., Xu, E. Y., Wang, Y.X., Chang, Y.F., \& Xiang, Q.L., “A Leaf Recognition Algorithm for Plant Classification Using Probabilistic Neural Network", IEEE 7th Interantional Symposium on Signal Processing and Information Technology, 2007.

10. Wu, Q., Zhou, C., \& Wang, C., "Feature Extraction and Automatic Recognition of Plant Leaf Using Artificial Neural Network", Avances en Ciencias de la Computacion, pp. 5-12, 2006. 